\title{
2020: a year of loss and delays
}

\author{
As the world came to a standstill in the spring of 2020, so did the work on our Focus issue on exercise metabolism \\ and health, which was originally scheduled for publication to coincide with the 2020 Summer Olympic Games.
}

ince the modern Olympic Games were fist held in 1896, they have never previously been delayed. The Summer Olympic Games were cancelled in 1916 because of World War I, and in 1940 and 1944 because of World War II. The first official postponement of the Olympics, from 2020 to 2021, is due to another very different kind of war: a war against a single-stranded-RNA virus, the coronavirus SARS-CoV-2, which has caused the COVID-19 pandemic. As everything came to a standstill in the spring of 2020 , so did the work on our Focus issue on exercise metabolism and health, which was originally scheduled for publication to coincide with the 2020 Olympics. Although it was delayed as SARS-CoV-2 dominated our lives, our Focus has now been published, with Review, Comment and research Articles revisiting foundational topics and highlighting new paradigms in exercise and muscle metabolism.

In the spirit of the Olympic Games, our first article is a Comment by Sarzynski and Bouchard on the determinative roles of genetics and genetic variants that, in concert with practice and training-the most important factors in Olympic performance-may predispose individuals to be able to achieve world-class athletic performance. A Review from Goodyear and colleagues discusses the effects of maternal and paternal exercise on offspring metabolic health in adulthood, the time in life when metabolic diseases typically surface. These remarkable beneficial effects of parental exercise are evident even before conception. Although the benefits of exercise on individuals are clear, understanding of the molecular triggers that orchestrate these beneficial effects remains incomplete and has been a topic of intense research in recent years. Wisløff and colleagues provide a comprehensive Review of the cardiac effects of physical activity, with particular focus on the physiological and molecular effects of exercise on healthy, diseased and aged hearts.

The second part of our Focus examines the more nuanced effects and adaptations of exercise. Exercise is a physiological state in which intertissue communication is of paramount importance in maintaining overall cellular, tissue and physiological homeostasis. Cellular metabolic demand skyrockets during intense exercise, thus rendering communication of the metabolic state essential for organismal homeostasis. Febbraio and colleagues, in their Review, discuss the physiological processes governing intertissue communication during exercise and the molecules mediating such cross-talk. We then dive deeper into the metabolic pathways that govern skeletal energy metabolism during exercise, with a Review by Hargreaves and Spriet, who describe regulatory mechanisms of ATP resynthesis during exercise and summarize nutritional interventions that target muscle metabolism and enhance athletic performance. Mounting experimental evidence has led to a general perception that the gut microbiome regulates anything and everything, including physiology, disease, signalling and metabolism. Chambers and colleagues provide a Review of emerging understanding of the mechanisms through which the three primary gut-derived short-chain fatty acids-acetate, propionate and butyrate-affect skeletal muscle metabolism and function, and highlight the roles of these gut-derived metabolites in skeletal muscle function and exercise capacity. Finally, Jones and colleagues, in their Review, highlight how exercise-induced alterations in the tumour microenvironment can affect immune cell function and how immune cells can mediate the benefits of exercise on cancer progression.

2020 is a lost year on many frontsincluding the loss of lives, the loss of jobs and the resultant economic slump, and the loss of certainty in a world we once knew-whose effects may be irreparable. During these challenging times, mandatory or voluntary isolation and social distancing have made exercise difficult for most people around the globe. For the poor and marginalized people worldwide who struggle to make ends meet, the beneficial effects of exercise may be a luxury entirely out of reach. The tragic circumstances that have led to the unprecedented delay of the 2020 Summer Olympics should serve as an opportunity for self-reflection for all of us. The COVID-19 pandemic and the ensuing isolation suspended many of our daily freedoms, opportunities and luxuries, such as exercise, but it did not insulate us from witnessing the struggles of those around the world. We see that the restlessness and boredom of isolation are incomparable to the hardships of those who have always struggled to survive. Similarly to how engaging in exercise can transform and improve our health, embracing humility, generosity and compassion instead of fear, scepticism and egotism could lead to a healthy, positive transformation for society beginning in 2020 .

Published online: 17 September 2020 https://doi.org/10.1038/s42255-020-00286-3 